\title{
DETERMINANTS ON SEMILATTICES
}

\section{BERNT LINDSTRÖM}

1. The following theorem contains Theorem 2 in [1] as a special case:

THEOREM. Let $X=\left\{x_{1}, x_{2}, \cdots, x_{n}\right\}$ be a semilattice with $\wedge$ as product and partially ordered such that $a \leqq b$ if and only if $a \wedge b=a$. Given functions $f_{i}(x), x \leqq x_{i}(x), i=1,2, \cdots, n$, with values in a commutative ring with unit, the following equality holds:

$$
\operatorname{det}\left(f_{i}\left(x_{i} \wedge x_{j}\right)\right)_{i, j=1}^{n}=\prod_{i=1}^{n} \sum_{j=1}^{n} f_{i}\left(x_{j}\right) \mu\left(x_{j}, x_{i}\right),
$$

where $\mu$ is the Möbius function of $X$.

Let $f_{i}\left(x_{j}\right)=\operatorname{sign} \mu\left(x_{j}, x_{i}\right)=1$ or -1 for $\mu\left(x_{j}, x_{i}\right) \geqq 0$ or $<0$ resp., then we get as a corollary

Corollary.

$$
\operatorname{det}\left(\operatorname{sign} \mu\left(x_{i} \wedge x_{j}, x_{i}\right)\right)_{i, j=1}^{n}=\prod_{i=1}^{n} \sum_{j=1}^{n}\left|\mu\left(x_{j}, x_{i}\right)\right| .
$$

This corollary can be applied to the construction of some $( \pm 1)$ determinants with large values.

For the background on generalized Möbius functions we refer to the paper [2] by Gian-Carlo Rota.

2. We first prove a lemma.

Lemma. Let $X$ be a finite-semilattice and $a, b \in X$ such that $b$ Then for a function $f(x), x \leqq a \wedge b$, with values in a commutative ring with unit, we get

$$
S=\sum_{x \leq b} f(x \wedge a) \mu(x, b)=0 .
$$

Proof. By a theorem of L. Weisner [2, p. 351], $\sum_{x \wedge a=b} \mu(x, 1)=0$ if $a<1$. We find, since $a \wedge b<b$,

$$
S=\sum_{y \leq a \wedge b} f(y) \sum_{x \wedge a=y} \mu(x, b)=\sum_{y \leq a \wedge b} f(y) \sum_{x \wedge a \wedge b=y} \mu(x, b)=0 .
$$

The restriction $x \leqq b$ is omitted, since $\mu(x, b)=0$ for $x \leq b$.

Received by the editors September 2, 1967. 
Proof of the Theorem. Permute indices for the elements in $X$ such that $x_{i}<x_{j}$ only if $i<j$. The value of the determinant is not changed when rows and columns are permuted in the same way. The matrix $M=\left(\mu\left(x_{i}, x_{j}\right)\right)_{i, j=1}^{n}$ is now triangular with 0 's below the main diagonal and 1's on it. Hence $\operatorname{det} M=1$. Define the matrix $N$ $=\left(f_{i}\left(x_{i} \wedge x_{j}\right)\right)_{i, j=1}^{n}$. It follows from the lemma that the product $N M$ is triangular with all elements 0 above the main diagonal. We find

$$
\operatorname{det} N=\operatorname{det} N M=\prod_{i=1}^{n} \sum_{j=1}^{n} f_{i}\left(x_{j}\right) \mu\left(x_{j}, x_{i}\right),
$$

and the theorem is proved.

We give a few examples:

Example 1. Let $X$ be a (closed) family of finite sets such that $N \in X$ and $M \subset N$ implies $M \in X . X$ is then a semilattice with $\wedge$ as the operation of taking intersections. By the principle of inclusionexclusion [2, p. 345], $\mu(M, N)=(-1)^{n(N)-n(M)}$ for $M \subset N(n(N)$ is the cardinality of $N)$. Theorem 2 in [ 1 ] follows easily.

Example 2. Let $X=\{1,2,3,5,30\}$ ordered by divisibility. From the Corollary we get a $( \pm 1)$-determinant of the order 5 with the value 48 , which is maximum for $( \pm 1)$-determinants of the order 5 (cf. [3, p. 82]). Let $Y=\{1,7\}$ and take the direct product of the lattices $X$ and $Y$. We get a lattice of the order 10 and then by the Corollary a $( \pm 1)$-determinant of the order 10 with the value $9 \cdot 2^{13}$, which is maximum for $( \pm 1)$-determinants of the order 10 .

By trial one easily determines lattices of the orders 12 and 13 and corresponding $( \pm 1)$-determinants with values $>1 / 3$ of the maximum for these determinants.

\section{REFERENCES}

1. B. Lindström and H.-O. Zetterström, A combinatorial problem in the k-adic number system, Proc. Amer. Math. Soc. 18 (1967), 166-170.

2. G.-C. Rota, On the foundations of combinatorial theory. I: Theory of Möbius functions, Z. Wahrscheinlichkeitstheorie und Verw. Gebiete. 2 (1964), 340-368.

3. M. Wojtas, On Hadamard's inequality for the determinants of order non-divisible by 4, Colloq. Math. 12 (1964), 73-83.

UNIVERSITY OF STOCKHOLM 